Positions moyennes des étoiles de comparaison.

\begin{tabular}{|c|c|c|c|}
\hline$*$ & $\alpha 1900.0$ & $\delta 1900.0$ & Autorité \\
\hline $\mathbf{I}$ & $9^{\mathrm{h}} 3 \mathrm{I}^{\mathrm{m}} 12^{\mathrm{s}} \cdot 2 \mathrm{I}$ & $+22^{\circ} 41^{\prime} 32^{\prime \prime} 9$ & AG. Berlin B. 3797 \\
\hline 2 & $\begin{array}{lll}9 & 27 & 34.37\end{array}$ & +2237 & AG. Berlin B. $379^{\circ}$ \\
\hline
\end{tabular}

Observatoire de Nice, 1900 mars 28.

\begin{tabular}{|c|c|c|c|}
\hline$*$ & $\alpha$ I 900.0 & $\delta: 900.0$ & Autorité \\
\hline $\begin{array}{l}3 \\
4\end{array}$ & $\begin{array}{lll}9^{\mathrm{h}} & 17^{\mathrm{m}} 3^{6 \mathrm{~s}} \cdot 5^{0} \\
9 & 12 & 54.67\end{array}$ & $\begin{array}{l}+22^{\circ} 36^{\prime} 23^{\prime \prime} \circ \\
+2 \text { I } 5^{8} 3^{6.8}\end{array}$ & $\begin{array}{l}\text { AG. Berlin B. } 3738 \\
\text { AG. Berlin B. } 3716\end{array}$ \\
\hline
\end{tabular}

A. Charlois.

\title{
Eléments et Ephéméride de la planète 1900 FA
}

calculés à l'aide des observations de Nice des 22 février, 5 et 22 mars 1900.

Epoque 1900 mars 22.5 t. m. Paris.

$$
\begin{aligned}
& M=296^{\circ} 57^{\prime} 42 !^{\prime \prime} 5 \\
& \omega=2173^{8} 48.2 \\
& \delta=\begin{array}{lll}
11 & 29 & 24.8
\end{array} 1900.0 \\
& i=\begin{array}{lll}
534 & 12.7
\end{array} \\
& \varphi=6^{\circ} 21^{\prime} 32.3 \\
& \mu=1098.58 \\
& \log a=0.33945^{\circ}
\end{aligned}
$$

\begin{tabular}{|c|c|c|c|c|c|c|c|}
\hline 1900 & \multicolumn{3}{|c|}{$\alpha$} & \multicolumn{2}{|r|}{$\delta$} & $\log r$ & $\log \Delta$ \\
\hline Mars 2 & $9^{h}$ & & $3^{\mathrm{s}}$ & $+21^{\circ}$ & $54: 7$ & 0.3213 & 0.1197 \\
\hline 2 & & & I 5 & 21 & 46.4 & & \\
\hline 2 & & & & 2 I & $37 \cdot 7$ & 0.3203 & 0. 1298 \\
\hline 3 & & & 10 & 21 & 28.4 & & \\
\hline Avril & & & 2 I & $2 \mathrm{I}$ & I 8.6 & 0.3192 & 0.1401 \\
\hline & & 9 & 40 & 2 I & 8.2 & & \\
\hline & & 10 & 8 & 20 & $57 \cdot 3$ & 0.3182 & 0.1508 \\
\hline & & Io & 44 & 20 & 45.9 & & \\
\hline & & I I & 28 & 20 & 34.1 & 0.3172 & 0.1616 \\
\hline $\mathbf{1}$ & & 12 & 20 & 20 & 21.8 & & \\
\hline I & 9 & I 3 & 20 & +20 & 9. I & 0.3162 & 0.1724 \\
\hline
\end{tabular}

\begin{tabular}{|c|c|c|c|c|c|c|}
\hline \multicolumn{2}{|c|}{1900} & $\alpha$ & \multicolumn{2}{|r|}{$\delta$} & $\log r$ & $\log \Delta$ \\
\hline \multicolumn{2}{|c|}{ Avril 13} & $9^{\mathrm{h}} 1^{\mathrm{m}} 20^{\mathrm{s}}$ & \multicolumn{2}{|c|}{$+20^{\circ} \quad 9^{\prime} .1$} & \multirow[t]{2}{*}{0.3162} & \multirow[t]{2}{*}{ O.I 724} \\
\hline & I 5 & 1427 & 19 & $5^{6.0}$ & & \\
\hline & 17 & $154 \mathrm{r}$ & I 9 & 42.4 & 0.3152 & $0.183^{2}$ \\
\hline & 19 & 17 & I 9 & 28.5 & & \\
\hline & 21 & 1828 & 19 & I 4.1 & 0.3142 & 0.1940 \\
\hline & 23 & $20 \quad 1$ & 18 & 59.4 & & \\
\hline & 25 & 2140 & 18 & $44 \cdot 3$ & 0.3132 & 0.2044 \\
\hline & 27 & 2326 & I 8 & 28.8 & & \\
\hline & 29 & $25 \quad 18$ & 18 & I 2.9 & 0.3122 & 0.2151 \\
\hline Mai & I & 27 I 5 & I 7 & 56.6 & & \\
\hline & 3 & $929 \times 7$ & +17 & 40.0 & $0.3 \times 13$ & 0.2254 \\
\hline
\end{tabular}

Grandeur: 1900 avril I 12.5 ; mai 3 12.9.

Observatoire de Nice, 1900 mars 28.

A. Charlois.

\section{Entdeckung eines neuen Planeten 1900 FC.}

Neuer Planet 1900 März 28 1 $2^{\mathrm{h}}{ }_{1} 8^{\mathrm{m}} .7$ M. Z. Heidelb. RA. $=191^{\circ} 8^{\prime}$ PD. $=90^{\circ} 4^{\prime}$ Gr. I1.2. Schwassmann.

Beobachtungen von Dr. $A$. Schwassmann in Heidelberg.

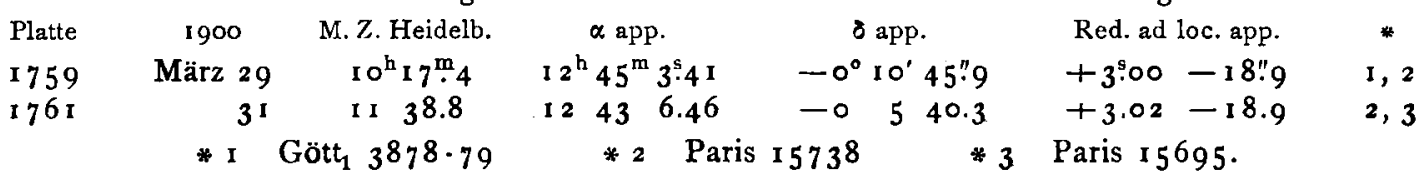

\section{Versammlung deutscher Naturforscher und Aerzte.}

Die diesjährige Versammlung deutscher Naturforscher und Aerzte, mit welcher die Jahresversammlung der deutschen Mathematiker-Vereinigung verbunden sein wird, wird vom 17. bis 22. September in Aachen stattinden. Die Anmeldung von Vorträgen und Demonstrationen wird bis Ende April erbeten. $K r$.

Planet (338) Budrosa. Corr. der Ephemeride (Veröff. R. I. Nr. I I) : April $2+\mathrm{I}^{\mathrm{m}} 4^{8^{\mathrm{s}}}-11^{\prime} 4$ Gr. $12^{\mathrm{m}}$. Palisa.

Inhalt zu Nr. 3634. 7. Palisa. Beobachtungen von Planeten, Cometen und Nebeln. 145. - Rambaud et Sy. Observations de comètes et de planètes. 153. - Th. D. Anderson. New Variable Star in Taurus. 155. - Th. D. Anderson. New Variable Star in Cassiopeia. 157. - E.F. Coddington. Observations of Comet 1898 VII. 157.- A. Charlois. Observations de la planète 1900 FA. 157. - A. Charlois. Eléments et Ephéméride de la planète 1900 FA. 159. - Entdeckung eines neuen Planeten I900 FC. I59. - 72. Versammlung deutscher Naturforscher und Aerzte. 159. - Palisa. Planet (338) Budrosa. 159. 